\title{
Trapped surfaces as boundaries for the constraint equations
}

\author{
Sergio Dain \\ Max-Planck-Institut für Gravitationsphysik \\ Am Mühlenberg 1 \\ 14476 Golm \\ Germany
}

September 29, 2018

\begin{abstract}
Trapped surfaces are studied as inner boundary for the Einstein vacuum constraint equations. The trapped surface condition can be written as a non linear boundary condition for these equations. Under appropriate assumptions, we prove existence and uniqueness of solutions in the exterior region for this boundary value problem. We also discuss the relevance of this result for the study of black holes collisions.
\end{abstract}

PACS: 04.20.Dw, 04.20.Ex, 04.70.Bw

\section{Introduction}

A black hole defines a boundary in the spacetime: the boundary of the region of no escape to infinity. This boundary is called the event horizon. There exist others kinds of spacetime boundaries, for example the ones defined by matter sources: the interface between matter and vacuum. In this case the boundary is introduced in the sources of Einstein equations by choosing an energy density with compact support. For black holes, the boundary is produced by the vacuum equations themselves, it depends only on fundamental properties of gravity; in this sense it is a more fundamental kind of boundary than the matter sources ones.

In the presence of a boundary, is natural to study boundary conditions for the equations and solve only for the exterior region. In the case of black 
holes this is very desirable since, firstly, the observations are made at infinity which is causally disconnected with the black hole region. And, secondly, in the black hole region there are singularities which are very difficult to handle in the numeric simulations.

In the context of an initial value formulation, the first step in order to understand the black hole boundary value problem is the study of the intersection of this boundary with a spacelike three-dimensional Cauchy hypersurface. That is, to study the black hole boundary value problem for the constraint equations. This will be the subject of this article.

There is no known way to characterize the intersection of the event horizon with a Cauchy surface in terms of a differential condition which involves only fields on the Cauchy surface. Such local characterization is provided by the concept of trapped surface. A trapped surface is a compact, twodimensional surface for which the expansions of both sets, outgoing and ingoing, of future directed null geodesics orthogonal to the surface are negative. The relevance of trapped surfaces relies in two important results: i) a trapped surface is always inside an event horizon. ii) The development of an initial data set which contain a trapped surface will be geodesically incomplete (see [26] and [38]). Because of i) we expect that trapped surfaces should be good boundaries for the constraint equations. In this article we will see that in fact this is true: trapped surfaces provide inner boundary conditions for the constraint equations under appropriate assumptions.

Associated with trapped surfaces there is the concept of an apparent horizon. An apparent horizon is essentially defined as the outermost trapped surface. In this article only trapped surfaces will be discussed and not apparent horizons, since with the techniques used here it will allows to decide whether a given trapped surface is in fact the outermost.

The problem of constructing initial data with several disconnected trapped surfaces is relevant for the study of black holes collisions (see for example [11] and [15]). In almost all data currently used in numerical simulations (the exceptions are the numerical studies in 36 and [28) trapped surfaces are produced by indirect means, for example introducing a non trivial topology. The theorem proved in this article will provide a way of solving only for the exterior region, with the appropriate boundary conditions, without imposing any symmetry and without introducing any non trivial topology.

From the point of view of getting initial data for black hole collisions this has several advantages. Firstly, no computer resources are wasted in the interior region. Secondly, the location of the trapped surfaces is known a priori. Finally, the control of the boundary conditions presented here will allow one to construct more general classes of black hole initial data than the ones studied so far (see the discussion in section 2). 
The plan of the article is the following. In section 2 we present our result, given by theorems 2.1] and 2.2, We also discuss the physical interpretation of these theorems. In section 3 we collect some results from the elliptic theory that will be used in the proofs. In section 4 and section 5 we prove theorem 2.1 and theorem 2.2 respectively.

\section{Main Result}

Let $C_{k}$ be a finite collection of compact sets in $\mathbb{R}^{3}$. We define the exterior region $\tilde{\Omega}=\mathbb{R}^{3} \backslash \cup_{k} C_{k}$. An initial data set for the Einstein vacuum equations is given by the triple $\left(\tilde{\Omega}, \tilde{h}_{a b}, \tilde{K}_{a b}\right)$ where $\tilde{h}_{a b}$ is a (positive definite) Riemannian metric, and $\tilde{K}_{a b}$ a symmetric tensor field on $\tilde{\Omega}$, such that they satisfy the constraint equations

$$
\begin{gathered}
\tilde{D}^{b} \tilde{K}_{a b}-\tilde{D}_{a} \tilde{K}=0, \\
\tilde{R}+\tilde{K}^{2}-\tilde{K}_{a b} \tilde{K}^{a b}=0,
\end{gathered}
$$

on $\tilde{\Omega}$; where $\tilde{D}_{a}$ is the covariant derivative with respect to $\tilde{h}_{a b}, \tilde{R}$ is the trace of the corresponding Ricci tensor, and $\tilde{K}=\tilde{h}^{a b} \tilde{K}_{a b}$.

The data will be called asymptotically flat if there exists some compact set $C$, with $\cup_{k} C_{k} \subset C$, such that $\tilde{\Omega} \backslash C$ can be mapped by a coordinate system $\tilde{x}^{j}$ diffeomorphically onto the complement of a closed ball in $\mathbb{R}^{3}$ and we have in these coordinates

$$
\begin{gathered}
\tilde{h}_{i j}=\left(1+\frac{2 m}{\tilde{r}}\right) \delta_{i j}+O\left(\tilde{r}^{-2}\right), \\
\tilde{K}_{i j}=O\left(\tilde{r}^{-2}\right),
\end{gathered}
$$

as $\tilde{r}=\left(\sum_{j=1}^{3}\left(\tilde{x}^{j}\right)^{2}\right)^{1 / 2} \rightarrow \infty$, where the constant $m$ is the total mass of the initial data.

The boundaries $\partial C_{k}$ are assumed to be smooth, two dimensional surfaces in $(\tilde{\Omega}, \tilde{h})$. Let $\tilde{\nu}^{a}$ be the unit normal of $\partial C_{k}$, with respect to $\tilde{h}_{a b}$, pointing in the outward direction of $\tilde{\Omega}$. Let $t^{a}$ be the unit timelike vector field orthogonal to the hypersurface $\tilde{\Omega}$ with respect to the spacetime metric $g_{a b}\left(t^{a} t^{b} g_{a b}=\right.$ -1 with our signature convention) The outgoing and ingoing null geodesics orthogonal to $\partial C_{k}$ are given by $l^{a}=t^{a}-\tilde{\nu}^{a}$ and $k^{a}=t^{a}+\tilde{\nu}^{a}$ respectively, the corresponding expansions are given by $\Theta_{+}=\nabla_{a} l^{a}$ and $\Theta_{-}=\nabla_{a} k^{a}$, where $\nabla_{a}$ is the connexion with respect to $g_{a b}$, see Fig. 11. We can calculate these 
expansions in terms of quantities intrinsic to the initial data

$$
\begin{aligned}
& \Theta_{-}=\tilde{K}+\tilde{H}-\tilde{\nu}^{a} \tilde{\nu}^{b} \tilde{K}_{a b}, \\
& \Theta_{+}=\tilde{K}-\tilde{H}-\tilde{\nu}^{a} \tilde{\nu}^{b} \tilde{K}_{a b} .
\end{aligned}
$$

where $\tilde{H}=\tilde{D}_{a} \tilde{\nu}^{a}$. Note that $\tilde{K}=\nabla_{a} t^{a}$ is the mean curvature of the threedimensional hypersurface $\tilde{\Omega}$ with respect to the spacetime metric $g_{a b}$ and the normal $t^{a}$, and $\tilde{H}$ is the mean curvature of the two-dimensional surface $\partial C_{k}$ with respect to the metric $\tilde{h}_{a b}$ and the normal $\tilde{\nu}^{a}$. Our choice of the definition of the extrinsic curvature is given by $\tilde{K}_{a b}=\tilde{h}_{a}^{c} \nabla_{c} t_{b}$ which agree with [38] but is the negative of the choice made in 33 . If $\partial C$ is an sphere of radius $\tilde{r}$ on a canonical Minkowski slice $t=$ const., then we have $\Theta_{+}=2 / \tilde{r}, \Theta_{-}=-2 / \tilde{r}$, $\tilde{H}=-2 / \tilde{r}, \tilde{K}_{a b}=0$, and $\tilde{v}^{a}=-(\partial / \partial \tilde{r})^{a}$.

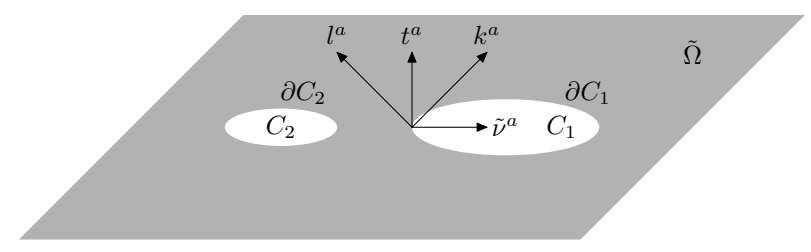

Figure 1: The exterior region $\tilde{\Omega}$ with two boundary components $\partial C_{1}$ and $\partial C_{2}$.

The boundary $\partial C_{k}$ will be called a future trapped surface if $\Theta_{+}<0$ and $\Theta_{-}<0$ on $\partial C_{k}$ and a future marginally trapped surface if $\Theta_{+} \leq 0$ and $\Theta_{-} \leq 0$.

We want to find solutions of the constraint equations (11)-(2) which are asymptotically flat (i.e; they satisfy (3)-(4)) and such that all the boundary components $\partial C_{k}$ (i.e; the whole boundary $\partial \tilde{\Omega}$ ) are trapped surfaces. In order to achieve this, we will reduce the constraint equations to an elliptic boundary value problem in which a negative (non-positive) ingoing null expansion $\Theta_{-}$ can, essentially, be freely prescribed at the boundary $\partial \tilde{\Omega}$. Under further restrictions on $\Theta_{-}$it will follows that also $\Theta_{+}$will be negative (non-positive), and hence the boundary will be future trapped (future marginally trapped). We emphasize that only $\Theta_{-}$(and not $\Theta_{+}$) will be free data on the boundary.

The elliptic reduction will be given by the conformal method. Also, instead of working with the exterior region $\tilde{\Omega}$ it will be more convenient to work with its related compactification $\Omega$. In the following we describe both procedures (see [9], 10] and the references therein for a description of the conformal method, and references [5], [20], [21], [16] for the compactification procedure). 


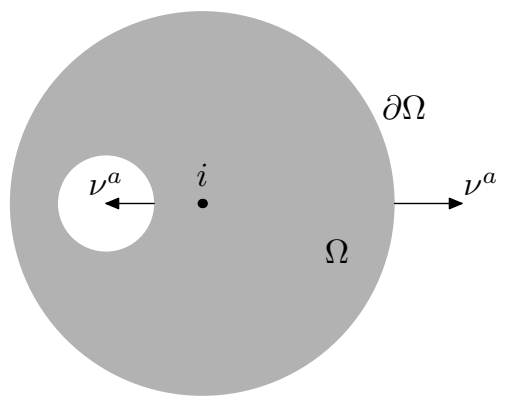

Figure 2: The compactification $\Omega$ of the exterior region $\tilde{\Omega}$ showed in Fig. $\square$

Let $\Omega$ a bounded domain in $\mathbb{R}^{3}$. We will assume that $\Omega$ is connected, however in general it will not be simply connected, each hole in $\Omega$ will correspond to an extra boundary component $\partial C_{k}$. Let $i \in \Omega$ be an arbitrary point and set $\tilde{\Omega}=\Omega \backslash\{i\}$. The point $i$ will represent the infinity of $\tilde{\Omega}$. Let $h_{a b}$ be a Riemannian metric defined on $\bar{\Omega}$. We denote by $\nu^{a}$ the unit normal to $\partial \Omega$ with respect to $h_{a b}$, pointing in the outward direction, see Fig. 22. We will assume that the conformal metric satisfies

$$
h_{a b} \in C^{\infty}(\bar{\Omega} \backslash\{i\}) \cap C^{2}(\bar{\Omega}) .
$$

We make different assumptions concerning the regularity of $h_{a b}$ at $\bar{\Omega} \backslash\{i\}$ and at $i$. The regularity assumption at $i$ is concerned with the fall off behavior of the solution at infinity, it is not related with the inner boundaries $\partial C_{k}$. The physical motivation for this distinction has been discussed in [17, [13] and [12]; in particular assumption (77) is weaker that the ones made in these references.

Let $K^{a b}$ be a symmetric, trace free tensor with respect to $h_{a b}$, such that

$$
D_{a} K^{a b}=0 \text { on } \tilde{\Omega},
$$

and let $\psi$ be a positive solution of

$$
L_{h} \psi=-\frac{1}{8} K_{a b} K^{a b} \psi^{-7} \text { on } \tilde{\Omega},
$$

where $L_{h} \equiv D^{a} D_{a}-R / 8$ and $R$ is the Ricci scalar of the metric $h_{a b}$. If we are able to find solutions of equations (8) -(9) , then the physical fields $(\tilde{h}, \tilde{K})$ defined by $\tilde{h}_{a b}=\psi^{4} h_{a b}$ and $\tilde{K}^{a b}=\psi^{-10} K^{a b}$ will satisfy equations (11)-(2) on $\tilde{\Omega}$. Since we have assumed $K=0$ we will obtain $\tilde{K}=0$.

We discuss now boundary conditions for equations (8) - (9). There are two kind of boundary conditions. The first one is asymptotic flatness, we want to 
ensure that the solution will satisfies the fall off (3)-(44). For this we require

$$
K^{a b}=O\left(r^{-4}\right) \text { at } i,
$$

and

$$
\lim _{r \rightarrow 0} r \psi=1 \text { at } i,
$$

where $x^{i}$ are normal coordinates with respect to the metric $h_{a b}$ centered at $i$ and $r$ the corresponding radius.

The second boundary condition is the requirement that $\Theta_{-}$should be freely prescribed at the boundary $\partial \Omega$. In order to write this condition we first calculate the null expansions $\Theta_{+}$and $\Theta_{-}$in term of the conformal quantities

$$
\begin{aligned}
& \Theta_{-}=\psi^{-3}\left(4 \nu^{a} D_{a} \psi+H \psi-\psi^{-3} K_{a b} \nu^{a} \nu^{b}\right), \\
& \Theta_{+}=\psi^{-3}\left(-4 \nu^{a} D_{a} \psi-H \psi-\psi^{-3} K_{a b} \nu^{a} \nu^{b}\right),
\end{aligned}
$$

where we have used that $K=\tilde{K}=0$, the normal vectors are related by $\tilde{\nu}^{a}=\psi^{-2} \nu^{a}$ (that is, $\nu^{a}$ is a unit vector with respect to the conformal metric $h_{a b}$ ) and $H=D_{a} \nu^{a}$. From equation (12) we deduce the following boundary condition for the conformal factor $\psi$

$$
N_{h} \psi=\psi^{3} \Theta_{-}+\psi^{-3} K_{a b} \nu^{a} \nu^{b} \text { on } \partial \Omega,
$$

where $N_{h} \equiv 4 \nu^{a} D_{a}+H$.

Equation (14) is a boundary condition for the conformal factor only. Remains to prescribe the boundary condition for the momentum constraint (8). From (14) we see that the simpler way of solving our problem will be to chose for the momentum constraint a boundary condition such that the function $K_{a b} \nu^{a} \nu^{b}$ can be freely prescribed on $\partial \Omega$. Our first result, concerning the momentum constraint, essentially says that this is possible. In order to write this theorem we need to introduce some additional concepts.

The conformal Killing operator $\mathcal{L}_{h}$, acting on vectors fields $w^{a}$, is defined as

$$
\left(\mathcal{L}_{h} w\right)_{a b}=2 D_{(a} w_{a)}-\frac{2}{3} h_{a b} D^{c} w_{c} .
$$

We say that $\xi^{a}$ is conformal Killing vector field if it satisfies $\mathcal{L}_{h} \xi=0$ on $\bar{\Omega}$. Given a conformal Killing vector field $\xi^{a}$ we define the conformal Killing data at the point $i$ by

$$
k_{a}=\frac{1}{6} D_{a} D_{b} \xi^{b}(i), S^{a}=\epsilon_{b c}^{a} D^{b} \xi^{c}(i), q^{a}=\xi^{a}(i), a=\frac{1}{3} D_{a} \xi^{a}(i) .
$$

Since $\Omega$ is connected, the integrability conditions for conformal Killing fields (cf. [39]) entail that these ten conformal Killing data at $i$ determine the field $\xi^{a}$ uniquely on $\Omega$. 
Because of the singular behavior of $K^{a b}$ at $i$ given by (10), it will convenient to write the results in terms of a further rescaled metric $h_{a b}^{\prime}$ for which the curvature vanished at $i$. The metric $h_{a b}^{\prime}$ can be explicitly computed as follows. Denote by $B_{\epsilon}$ the open ball with center $i$ and radius $r=\epsilon>0$, where $\epsilon$ is chosen small enough such that $B_{\epsilon}$ is a convex normal neighborhood of $i$. We define the cut-off function $\chi_{\epsilon}$ as a non-negative, smooth function such that $\chi_{\epsilon}=1$ in $B_{\epsilon / 2}$ and $\chi_{\epsilon}=0$ in $\Omega \backslash B_{\epsilon}$. Consider the following smooth conformal factor

$$
\omega_{0}=e^{\chi_{\epsilon} f_{0}} \text { with } f_{0}=\frac{1}{4} x^{j} x^{k} L_{j k}(i),
$$

where we have used the value at $i$ of the tensor $L_{a b} \equiv R_{a b}-\frac{1}{4} R h_{a b}$. By a straightforward calculation we get that the Ricci tensor of the metric

$$
h_{a b}^{\prime}=\omega_{0}^{4} h_{a b}
$$

vanishes at the point $i$.

Equation (8) is solved using the standard York splitting (cf. [40]) adapted to our setting. The free data is a trace free, symmetric, tensor $Q^{a b}$. Since we have two kinds of boundary conditions, $Q^{a b}$ has a natural decomposition

$$
Q^{a b}=Q_{s i n g}^{a b}+Q_{r e g}^{a b},
$$

where $Q_{s i n g}^{a b}$ and $Q_{r e g}^{a b}$ can be roughly characterized as follows (see section 4 for details).

The tensor $Q_{s i n g}^{a b}$ is different from zero only in $B_{\epsilon}$ and pick up the singular behavior of $K^{a b}$ at $i$, i.e; it will blow up like $r^{-4}$ at $i$. It contains the linear and angular momentum of the data. These physical quantities appear as constants $P^{a}$ and $J^{a}$ which partially characterize $Q_{\text {sing }}^{a b}$. In addition to $P^{a}$ and $J^{a}$, there are other constants $Q^{a}$ and $A$ involved in $Q_{\text {sing }}^{a b}$; these ten constants are related with the conformal Killing data (16) as we will see.

The tensor $Q_{r e g}^{a b}$ gives the boundary value of $K^{a b}$ at $\partial \Omega$ and will not contribute to the linear and angular momentum. For example we can take $Q_{r e g}^{a b}$ smooth in $\bar{\Omega}$; however a more general behavior at $i$ is allowed, in particular $Q_{r e g}^{a b}$ can blow up like $r^{-1}$ at $i$.

With these definitions, we can write our existence theorem for the momentum constraint.

Theorem 2.1. Assume $h_{a b}$ satisfies (7) and $\partial \Omega$ is smooth. Let $Q^{a b}$ the symmetric, trace free tensor, given by (19) where $Q_{\text {reg }}^{a b}$ and $Q_{\text {sing }}^{a b}$ satisfy (63)(64) and (60) respectively. 
i) If the metric $h_{a b}$ admits no conformal Killing fields on $\Omega$, then there exists a unique vector field $w^{a} \in C^{\infty}(\bar{\Omega} \backslash\{i\}) \cap H^{1}(\Omega)$ such that the tensor $K^{a b}$ defined by

$$
K^{a b}=\omega_{0}^{10}\left(Q^{a b}-\left(\mathcal{L}_{h^{\prime}} w\right)^{a b}\right),
$$

satisfies the equation $D_{a} K^{a b}=0$ in $\tilde{\Omega}$ and $K^{a b} \nu_{a}=Q_{r e g}^{a b} \nu_{a}$ on $\partial \Omega$. The metric $h_{a b}^{\prime}$ and the conformal factor $\omega_{0}$ are defined by (18) and (17).

ii) If the metric $h_{a b}$ admits conformal Killing fields $\xi^{a}$ on $\Omega$, a vector field $w^{a}$ as specified above exists if and only if the constants $P^{a}, J^{a}, A$ and $Q^{a}$ (partly) characterizing the tensor field $Q_{\text {sing }}^{a b}$, satisfy the equation

$$
P^{a} k_{a}+J^{a} S_{a}+A a+\left(P^{c} L_{c}{ }^{a}(i)+Q^{a}\right) q_{a}=\int_{\partial \Omega} Q_{r e g}^{a b} \xi_{a} \nu_{b} d S,
$$

for any conformal Killing field $\xi^{a}$ of $h_{a b}$; where the constants $k^{a}, S^{a}$, a and $q^{a}$ are the conformal Killing data at $i$ for $\xi^{a}$ given by (16).

In both cases i) and ii) $K^{a b}$ is unique, $K^{a b} \in C^{\infty}(\bar{\Omega} \backslash\{i\})$ and $K^{a b}=O\left(r^{-4}\right)$ at $i$.

Note that in case ii) $K^{a b}$ is unique but $w^{a}$ is not, we can add to a given solution any conformal Killing vector. Condition (21), which arise in the presence of conformal symmetries, is the natural extension to the analog condition discussed in [6] and [16] where there is no inner boundary and in [17 where there exists matter sources in a compact region. The fact that not only Killing vectors but also conformal Killing vectors appear in (21) is a particular feature of maximal slices (i.e; $\tilde{K}=K=0$ ), see the discussion in 14.

The next result is concerning the Hamiltonian constraint (91). In order to enunciate it, we need two auxiliary solutions. The first one, denoted by $\psi_{0}$, is a solution of the problem with $K^{a b}=0$ on $\Omega$ (i.e; time symmetry) and $\Theta_{+}=\Theta_{-}=0$ on $\partial \Omega$. That is, $\psi_{0}$ satisfies the following linear boundary value problem

$$
\begin{aligned}
L_{h} \psi_{0} & =0 \text { in } \tilde{\Omega}, \\
N_{h} \psi_{0} & =0 \text { on } \partial \Omega, \\
\lim _{r \rightarrow 0} r \psi_{0} & =1 \text { at } i .
\end{aligned}
$$

The second particular solution, $\psi_{1}$, is a solution of the following linear boundary value problem

$$
\begin{aligned}
L_{h} \psi_{1} & =-\frac{1}{8} K_{a b} K^{a b} \psi_{0}^{-7} \text { in } \tilde{\Omega}, \\
N_{h} \psi_{1} & =\psi_{0}^{-3} K_{a b} \nu^{a} \nu^{b} \text { on } \partial \Omega, \\
\lim _{r \rightarrow 0} r \psi_{1} & =1 \text { at } i .
\end{aligned}
$$


Theorem 2.2. Assume that the conformal metric $h_{a b}$ satisfies (7) and that $\partial \Omega$ is smooth. Assume also that $R \geq 0$ in $\bar{\Omega}, H \geq 0$ on $\partial \Omega$ and that either $R$ or $H$ is not identically zero. Then:

i) There exist a unique, positive, solution $\psi_{0}$ of (22)-(24), and $\psi_{0} \in$ $C^{\infty}(\bar{\Omega} \backslash\{i\})$.

ii) Let $K^{a b}$ be given by theorem [2.1. Assume, in addition, that

$$
Q_{r e g}^{a b} \nu_{a} \nu_{b} \geq 0 \text { on } \partial \Omega .
$$

Then there exist a unique, positive, solution $\psi_{1}$ of (25)-(27), and $\psi_{1} \in$ $C^{\infty}(\bar{\Omega} \backslash\{i\})$.

iii) Let $\Theta_{-} \leq 0$ and assume it satisfies the following inequality

$$
\left|\Theta_{-}\right| \leq \psi_{1}^{-6} Q_{r e g}^{a b} \nu_{a} \nu_{b} .
$$

Then there exist a unique, positive, solution $\psi$ of equation (9) with boundary conditions (111) and (14), and $\psi \in C^{\infty}(\bar{\Omega} \backslash\{i\})$. Moreover, $\psi_{0} \leq \psi \leq \psi_{1}$ and

$$
\Theta_{+} \leq \Theta_{-} \leq 0 \text {. }
$$

Let us discuss the assumptions and the conclusions of this theorem. We have assumed that the boundary $\partial \Omega$ is smooth, this is done for simplicity and because there seems not be any physical reason to study trapped surfaces with rough boundary. For rough boundaries (for example with corners or cusps) the following proofs are not valid.

The condition $R \geq 0$ and $D_{a} \nu^{a} \geq 0$ ensures that the conformal metric $h_{a b}$ can be conformally rescaled to a metric $\hat{h}_{a b}$ for which the boundary $\partial \Omega$ is an extremal surface (i.e; $\hat{H}=0$ ) and $\hat{R}>0$. Then, this condition can expressed in terms of the corresponding Yamabe class studied in [18. A similar condition also appears in the case without inner boundary, see [7] and also [21], [16] for a discussion of the same condition using a compactification as we use here.

Simple examples of sets $(h, \Omega)$ which satisfy these conditions are the followings. Take $h_{a b}=\delta_{a b}$, where $\delta_{a b}$ is the flat metric. Let $\Omega=B_{r_{0}}$, where $B_{r_{0}}$ is a ball of radius $r_{0}$. Then $H=2 / r_{0}$ and $R=0$. This example has only one boundary component. Note that, in this example, we can not take a second ball inside $B_{r_{0}}$ to construct a region like the one showed in Fig. 2. because in this case on the boundary of the interior ball $H$ will be negative, owing to our choice of the normal $\nu^{a}$. To construct an example with two boundary components, take $h_{a b}=\omega^{4} \delta_{a b}$, where $\omega=1+1 / r$; and let $\Omega$ be the annulus $\Omega=B_{r_{0}}-B_{r_{1}}$ where $r_{0}>1>r_{1}>0$. Then we have that $R=0, H=2\left(1+1 / r_{0}\right)^{-3}\left(1-1 / r_{0}\right) / r_{0}>0$ on $\partial B_{r_{0}}$ and 
$H=2\left(1+1 / r_{1}\right)^{-3}\left(1 / r_{1}-1\right) / r_{1}>0$ on $\partial B_{r_{1}}$. Note that in this example the point $i$ can not be at the origin $r=0$. The generalization to $k$ boundaries components is straightforward.

Condition (28) can be written in terms of physical quantities as $\tilde{K}^{a b} \tilde{\nu}_{a} \tilde{\nu}_{b} \geq$ 0 . From equations (5)-(6) we see that, remarkably, every future marginally trapped surface on a maximal slice satisfies this condition. In contrast, condition (29) is a sufficient condition which is not expected to be also necessary in general.

A future trapped surface is a time asymmetric concept. If we reverse the time direction $t^{a} \rightarrow-t^{a}$ we have $l^{a} \rightarrow-k^{a}$ and $k^{a} \rightarrow-l^{a}$ and hence $\Theta_{+} \rightarrow-\Theta_{-}, \Theta_{-} \rightarrow-\Theta_{+}$. Then, if we have a future trapped surface in one time direction, we will have $\Theta_{+}, \Theta_{-}>0$ in the opposite time direction and then it will be not trapped in this time direction. The singularity theorems 35. implies that the development of a data which contain a future trapped surface will be geodesically incomplete into the future, but nothing is said about the past. In fact, in a realistic gravitational collapse, the spacetime is expected to have no singularities in the past. On the other hand, if the data have non-trivial topology (as the ones usually used in numerical simulations, see for example [11) we can apply the singularity theorem given in 22 to conclude that the spacetime will be geodesically incomplete in both time directions. This shows that the class of data constructed here is more general that the one with non-trivial topology, since it does not rule out data with regular past.

It is perhaps surprising that $\Theta_{-}$and not $\Theta_{+}$is free data at the boundary. For example, we can not prescribe $\Theta_{+}=0$ and $\Theta_{-}<0$ at the boundary, since this will contradict inequality (30). If we start with a boundary that satisfies $\Theta_{+}, \Theta_{-}<0$, then, in general, a surface with $\Theta_{+}=0, \Theta_{-}<0$ will be present in the interior of $\tilde{\Omega}$, this will correspond, for example, to the outer most trapped surface. But we can not use theorem 2.2 to start with such a surface at the boundary, the location of this surface can not be given a priori under our assumptions. On the other hand, theorem 2.2 allows to prescribe $\Theta_{+}=0$ at the boundary, this is just the time inversion of the case $\Theta_{-}=0, \Theta_{+} \leq 0$ which gives $\Theta_{+}=0, \Theta_{-} \geq 0$. However, unless $\Theta_{-}=0$, these surfaces will not be future marginally trapped. Moreover, these surfaces are located on the inner null boundary of the left quadrant of the Kruskal diagram (region IV of Fig. 6.9 in [38]), and hence they are not expected to be present in any realistic gravitational collapse of matter.

In the Kruskal diagram, trapped surfaces which satisfy condition (30) are located in the left half of region II. Hence, they can be present in a spherically symmetric collapse. However, condition (30) is a restriction in the sense that not in every collapse such surfaces will exist, as can be shown using the 
spherically symmetric example.

The choice of $\Theta_{-}$as a free data is dictated by the signs in the boundary condition (14). The boundary condition should have the appropriate signs to use the maximum principle in order to prove that the conformal factor $\psi$ is positive (see sections 3 and [5).

Finally, we note that theorems 2.1 and 2.2 show how to obtain the whole solution in the exterior region $\tilde{\Omega}$ solving the equations in the compact domain $\Omega$. This can be used in numerical computations to solve for the whole data on a finite grid (see for example [28], [29]). ${ }^{1}$

\section{Preliminaries}

In this section we summarize some results from functional analysis and the theory of linear elliptic partial differential equations which we will use in the following proofs. Some of these results, although standard, are not easily available in the literature.

The operators $L_{h}$ (defined after Eq. (91)) and $\mathbf{L}_{h}$ (defined by (48) ) which appear in the Hamiltonian and Momentum constraints respectively, are linear, second order, elliptic operators in divergence form. We will use the Hilbert space approach to the boundary value problem for this kind of operators. This approach has the advantage to be both simpler and applicable to a broader classes of solutions than other methods.

Let $\Omega$ a bounded open domain in $\mathbb{R}^{3}$, it will be assumed in the following that the boundary $\partial \Omega$ is smooth. We shall use the following functions spaces (see 1], 24] for definitions, notations, and results) defined in $\Omega$ : the set of $m$ times continuously differentiable functions $C^{m}(\Omega)$, the Hölder space $C^{m, \alpha}(\Omega)$, where $0<\alpha<1$, the corresponding spaces $C^{m}(\bar{\Omega}), C^{m, \alpha}(\bar{\Omega})$, the space $C_{0}^{\infty}(\Omega)$ of smooth function with compact support in $\Omega$, the Lebesgue space $L^{p}(\Omega)$, the Sobolev space $H^{s}(\Omega)$, and the local Sobolev space $H_{l o c}^{s}(\Omega)$ where $s$ is a real number.

An elliptic operator in divergence form has an associated bilinear form $\mathbf{B}$.

\footnotetext{
${ }^{1}$ After this work was completed, there has appeared an article by D. Maxwell [32] which studies the boundary condition $\Theta_{+}=0$. In a new version of this article, which appeared after this work was submitted for publication, Maxwell makes an important improvement and he is now able to construct solutions which satisfy $\Theta_{-} \leq \Theta_{+}=0$ at the boundary. This is done imposing an extra condition which involves $H$ and $K_{a b} \nu^{a} \nu^{b}$. Remarkably, the solutions obtained in 32 and the ones obtained in the present article do not, in general, overlap. However, it is important to emphasize that both set of solutions do not cover all possible black hole exterior regions. It is still an open problem how to construct and characterize all initial data for black holes exterior regions.

I am grateful to D. Maxwell for useful discussions.
} 
In the particular cases of $L_{h}$ and $\mathbf{L}_{h}$ these bilinear forms are given by (37) and (45) respectively. A bilinear form is called weakly coercive over $H^{1}(\Omega)$ if there exist constants $\lambda_{1}>0$ and $\lambda_{0} \geq 0$ such that following inequality holds

$$
\mathbf{B}(v, v) \geq \lambda_{1}\|v\|_{H^{1}(\Omega)}^{2}-\lambda_{0}\|v\|_{H^{0}(\Omega)}^{2}
$$

for all $v \in H^{1}(\Omega)$. If we can take $\lambda_{0}=0$ in (31) we say that $\mathbf{B}$ is strictly coercive. B is called bounded in $H^{1}(\Omega)$ if there exist a positive constant $C$ such that

$$
|\mathbf{B}(v, u)| \leq C|| v\left\|_{H^{1}(\Omega)}\right\| u \|_{H^{1}(\Omega)},
$$

for all $u, v \in H^{1}(\Omega)$. For a given $\mathbf{B}$, we define the following spaces

$$
\begin{gathered}
\mathcal{V}=\left\{u \in \mathcal{X}: \mathbf{B}(v, u)=0 \text { for all } v \in H^{1}(\Omega)\right\} \\
\mathcal{W}=\left\{u \in \mathcal{X}: \mathbf{B}(u, v)=0 \text { for all } v \in H^{1}(\Omega)\right\}
\end{gathered}
$$

When $\mathbf{B}$ is symmetric we, of course, have $\mathcal{V}=\mathcal{W}$.

The following theorem is the basic existence tool for the linear boundary problem, it is a consequence of the Lax-Milgram theorem and the Fredholm alternative in Hilbert spaces.

Theorem 3.1. Let $\mathbf{B}$ be a bilinear form which is bounded and weakly coercive in $H^{1}(\Omega)$. Then $\mathcal{V}$ and $\mathcal{W}$ have both finite dimension. Moreover, let $F$ be a bounded linear functional over $H^{1}(\Omega)$. Then, there exist a function $u \in H^{1}(\Omega)$ such that

$$
\mathbf{B}(v, u)=F(v) \text { for all } v \in H^{1}(\Omega),
$$

if and only if

$$
F(w)=0,
$$

for all $w \in \mathcal{W}$.

We will apply this theorem for both second order elliptic equations and second order elliptic systems. Although the methods in both cases are, in many aspect, similar, second order elliptic equations have distinguished properties, we will treat them separately.

Consider the operator $L_{h} u$. If we multiply by $v \in C_{0}^{1}(\Omega)$ and integrate by parts we obtain the symmetric bilinear form

$$
\mathbf{B}(u, v)=\int_{\Omega}\left(h^{a b} D_{a} u D_{b} v-R u v\right) d \mu,
$$

where $d \mu$ is the volume element with respect to $h_{a b}$. We have the following result, which will allow us to use theorem 3.1 for the existence proof. 
Theorem 3.2. Let $\mathbf{B}$ be given by (37). Assume that the metric satisfies (7). Then $\mathbf{B}$ is bounded and weakly coercive in $H^{1}(\Omega)$.

For a proof see for example [24], in [30] and [8] the same result is proved under more general assumptions on the coefficients.

Consider the following linear boundary value problem for the elliptic operator $L_{h}$

$$
\begin{aligned}
& L_{h} u=f \text { in } \Omega, \\
& N_{h} u=\varphi \text { on } \partial \Omega .
\end{aligned}
$$

Note that our assumption on $h_{a b}$ implies that $H \in C^{\infty}(\partial \Omega)$. A weak solution of this problem is a function $u \in H^{1}(\Omega)$ which satisfies

$$
\mathbf{B}(u, v)+\int_{\partial \Omega} H u v d S=-\int_{\Omega} f v d \mu+\int_{\partial \Omega} \varphi v d S,
$$

for all $v \in H^{1}(\Omega)$, where $\mathbf{B}$ is given by (37) and $d S$ is the surface element on $\partial \Omega$. One can check that a smooth weak solution $u$ of (40) will satisfies (38) - (39). This kind of boundary conditions are called natural boundary condition for the operator $L_{h}$, see [2], [30] and [19] for a discussion on this method of treating general boundary problem.

We define the bilinear form $\mathbf{B}^{\prime}$ by

$$
\mathbf{B}^{\prime}(u, v)=\mathbf{B}(u, v)+\int_{\partial \Omega} H u v d S,
$$

and the linear functional $F$ given by

$$
F(v)=-\int_{\Omega} f v d \mu+\int_{\partial \Omega} \varphi v d S .
$$

That is, $\mathbf{B}^{\prime}$ and $F$ are defined as the left and right hand side of Eq. (40) respectively. We want to apply the abstract existence theorem 3.1 for $\mathbf{B}^{\prime}$ and $F$. This theorem is not very useful unless we have a characterization of the null space $\mathcal{W}$ of $\mathbf{B}^{\prime}$. The maximum principle will be used to ensure that $\mathcal{W}$ is trivial under additional assumptions on the coefficients.

We have the following version of the maximum principle.

Theorem 3.3 (Weak Maximum Principle). Let $\mathbf{B}^{\prime}$ given by (41), where the metric satisfies (17). Assume that $R \geq 0$ and $H \geq 0$. Let $u \in H^{1}(\Omega)$ satisfies $\mathbf{B}^{\prime}(u, v) \leq(\geq) 0$ for all $v \in H^{1}(\Omega), v \geq 0$. Then $u \leq(\geq) 0$ in $\Omega$ or $u$ is a positive (negative) constant. 
This theorem can be obtained from the more general results given in [37. The proof is similar to the proof of theorem 8.1 in [24].

We will also use the following standard versions of the maximum principle, see [24].

Theorem 3.4 (Strong Maximum Principle). Assume that the metric satisfies (77) and that $R \geq 0$. Let $u \in H^{1}(\Omega)$ satisfy $L_{h} u \geq 0$ in $\Omega$. Then, if for some ball $B \subset \subset \Omega$ we have

$$
\sup _{B} u=\sup _{\Omega} u \geq 0,
$$

the function $u$ must be constant in $\Omega$.

Theorem 3.5 (Hopf). Assume that the metric satisfies (7) and that $R \geq 0$. Let $u \in C^{2}(\Omega)$. Suppose $L_{h} u \geq 0$ in $\Omega$. Let $x_{0} \in \partial \Omega$ be such that

(i) $u$ is continuous at $x_{0}$;

(ii) $u\left(x_{0}\right)>u(x)$ for all $x \in \Omega$ and $u\left(x_{0}\right) \geq 0$;

(iii) $\partial \Omega$ satisfies an interior sphere condition at $x_{0}$.

Then the outer normal derivative of $u$ at $x_{0}$, if it exist, satisfies the strict inequality

$$
\frac{\partial u}{\partial \nu}>0
$$

We can now prove the basic existence result for the elliptic linear boundary value problem (40).

Theorem 3.6. Assume that the metric satisfies (7). Assume also that $R \geq$ $0, H \geq 0$ and that either $R$ or $H$ is not identically zero. Let $f \in L^{2}(\Omega)$, $\varphi \in L^{2}(\partial \Omega)$. Then there exist a unique weak solution $u \in H^{1}(\Omega)$ of the boundary value problem (40). If, in addition, we have $f \leq 0, \varphi \geq 0$, then $u \geq 0$.

Proof. Let $\mathbf{B}^{\prime}$ and $F$ be given by (41) and (42). In order to apply theorem 3.1. we need to prove that $F$ is bounded, $\mathbf{B}^{\prime}$ coercive and bounded and finally that the corresponding null space $\mathcal{W}$ for $\mathbf{B}^{\prime}$ is trivial. The boundedness of $F$ follows directly from Hölder inequality and the assumptions on $f$ and $\varphi$.

By theorem 3.2 we have that $\mathbf{B}$ is bounded, then to prove that also $\mathbf{B}^{\prime}$ is bounded we only need to prove that the surface integral in (41) is bounded in $H^{1}(\Omega)$. Using the generalized Hölder inequality we obtain

$$
\left|\int_{\partial \Omega} H u v d S\right| \leq\|H\|_{L^{2}(\partial \Omega)}|| u\left\|_{L^{4}(\partial \Omega)}|| v\right\|_{L^{4}(\partial \Omega)} .
$$


Since $u, v \in H^{1}(\Omega)$, we can use the trace theorem (see [1]) to conclude that $u, v \in H^{1 / 2}(\partial \Omega)$, by the imbedding theorem we have that $u, v \in L^{4}(\partial \Omega)$. Then, we can replace the $L^{4}(\partial \Omega)$ norms in the left hand side of (44) by $H^{1}(\Omega)$ norms and hence the surface integral is bounded in $H^{1}(\Omega)$.

Using that $H \geq 0$ we have that $\mathbf{B}^{\prime}(v, v) \geq \mathbf{B}(v, v)$, then the coerciveness of $\mathbf{B}^{\prime}$ follows directly from the coerciveness of $\mathbf{B}$ given by theorem 3.2 .

From the maximum principle 3.3 it follows that every $w \in \mathcal{W}$ should be constant, by the assumption that either $R$ or $H$ is not identically zero it follows that $w=0$. The last statement about positivity of $u$ follows directly from the maximum principle 3.3 .

We note that if we make the stronger assumption $H>0$ we don't need to use the maximum principle in the previous proof, the uniqueness followed directly in this case.

The following global estimates for the non homogeneous case will play a fundamental role in the non linear existence proof.

Theorem 3.7. Assume that the metric satisfies (17). Let $u \in H^{1}(\Omega)$ be a weak solution of (40). Assume also that $f \in L^{2}(\Omega)$ and $\varphi \in L^{\infty}(\partial \Omega)$. Then $u \in C^{\alpha}(\bar{\Omega}), 0<\alpha<1$.

The interior estimate part of this theorem (that is the fact that $u \in$ $\left.C^{\alpha}(\Omega)\right)$ is standard. The crucial part is the regularity up to the boundary under the assumption $\varphi \in L^{\infty}(\partial \Omega)$. This can be proved using Campanato spaces, following similar arguments as in [23] and Theorem 3.8 of [25], where the interior estimate is proved.

We turn now to the elliptic system defined by $\mathbf{L}_{h} w^{a}$. If we multiply by a $u^{a} \in C_{0}^{\infty}(\Omega)$ we get the following bilinear form defined over vectors

$$
\mathbf{B}(u, w)=\int_{\Omega}\left(\mathcal{L}_{h} w\right)^{a b}\left(\mathcal{L}_{h} u\right)_{a b} d \mu .
$$

We have the following analog to theorem 3.2 .

Theorem 3.8. Assume that the metric satisfies (7). Then $\mathbf{B}$ defined by (45) is bounded and weakly coercive on $H^{1}(\Omega)$.

To prove this result, we first check that $\mathbf{B}$ satisfies the Legendre-Hadamard conditions and then we use the Gårding inequality for functions with compact support. Using the assumption of the smoothness of $\partial \Omega$ we can extend the result to $H^{1}(\Omega)$ using the Sobolev extension theorems. See for example 23. for a general discussion of this kind of bilinear forms.

Finally, for the non linear existence proof we will use the following fixed point theorem (see for example [24]). 
Theorem 3.9 (Schauder fixed point). Let $B$ be a closed convex set in a Banach space $V$ and let $T$ be a continuous mapping of $B$ into itself such that the image $T(B)$ is precompact, i.e. has compact closure in $B$. Then $T$ has a fixed point.

\section{The Momentum Constraint}

The aim of this section is to prove theorem 2.1. The momentum constraint is solved using the York splitting as follows. Given a trace free tensor $Q^{a b}$ we look for a vector field $w^{a}$ such that the tensor $K^{a b}$ defined by

$$
K^{a b}=Q^{a b}-\left(\mathcal{L}_{h} w\right)^{a b}
$$

satisfies (8). Then, it follows that the vector $w^{a}$ will satisfy the equation

$$
\mathbf{L}_{h} w_{a}=D^{b} Q_{a b} \text { in } \tilde{\Omega}
$$

where

$$
\mathbf{L}_{h} w_{a}=D^{b}(\mathcal{L} w)_{a b} .
$$

We want to prescribe $K^{a b} \nu_{a} \nu_{b}$ freely at the boundary $\partial \Omega$, this suggests to impose the following boundary condition for equation (48)

$$
(\mathcal{L} w)_{a b} \nu^{a}=0 \text { on } \partial \Omega
$$

where $\mathcal{L}_{h}$ has been defined in (15). This boundary condition satisfies the Lopatinski-Shapiro conditions required in the elliptic estimates of [3]. Moreover, this is a natural boundary condition for the operator $\mathbf{L}_{h}$ in the following sense. Assume that $u^{a}$ and $w^{a}$ are sufficiently smooth vector fields, we use (48) and integrate by part to obtain the following identity

$$
\int_{\Omega} u_{a} \mathbf{L}_{h} w^{a} d \mu=\int_{\partial \Omega}\left(\mathcal{L}_{h} w\right)^{a b} \nu_{a} u_{b} d S-\mathbf{B}(u, w)
$$

where the bilinear form $\mathbf{B}(u, w)$ is given by (45). From equation (50) we see that if $\mathbf{L}_{h} w^{a}=0$ in $\Omega$ and $(\mathcal{L} w)_{a b} \nu^{a}=0$ on $\partial \Omega$, then $w^{a}$ is a conformal Killing vector.

For the moment let us not consider the singular behavior at $i$. Then we have the following boundary value problem

$$
\begin{aligned}
\mathbf{L}_{h} w^{a} & =J^{a} \text { in } \Omega, \\
\left(\mathcal{L}_{h} w\right)_{a b} \nu^{a} & =0 \text { on } \partial \Omega .
\end{aligned}
$$


A vector field $w \in H^{1}(\Omega)$ is a weak solution of the boundary value problem (51)-(52) if

$$
\mathbf{B}(u, w)=-\int_{\Omega} u_{a} J^{a} d \mu,
$$

for all $u^{a} \in H^{1}(\Omega)$. Consistently with (53), we define a conformal Killing vector field $\xi^{a}$ as weak solution of

$$
\mathbf{B}(u, \xi)=0,
$$

for all $u^{a} \in H^{1}(\Omega)$. We have the following existence theorem.

Theorem 4.1. Assume that the metric satisfies (7). Let $J^{a} \in L^{p}(\Omega), p \geq$ $6 / 5$ be a vector field such that

$$
\int_{\Omega} J^{a} \xi_{a} d \mu=0
$$

for all conformal Killing vectors $\xi^{a}$ in $\Omega$. Then there exist a weak solution $w^{a} \in H^{1}(\Omega)$ of (53).

Proof. We will use theorem 3.1. By theorem 3.8 we have that the bilinear form $\mathbf{B}$ defined by (45) is bounded and coercive in $H^{1}(\Omega)$ under our assumptions on the metric $h_{a b}$. Using Hölder inequality and the Sobolev imbedding theorem one can prove that the functional

$$
F(u)=\int_{\Omega} u_{a} J^{a} d \mu,
$$

is bounded in $H^{1}(\Omega)$ if $J^{a} \in L^{p}(\Omega), p \geq 6 / 5$. Finally, condition (55) is just equation $F(\xi)=0$ in theorem 3.1

The boundary value problem (51)-(52) has been studied in elasticity, usually under stronger regularity assumptions on the coefficients (see for example [31]).

We analyze now the singular behavior at the point $i$. As we point out before, it will be convenient to take advantage of the conformal invariance of the equations and to write them with respect to the metric (18). Since the Ricci tensor of the metric (18) vanishes at $i$ and we are in three dimensions, the Riemann tensor vanishes there too. Hence the connection and metric coefficients of (18) satisfy in normal coordinates $x^{\prime k}$ (with respect to $h_{a b}^{\prime}$ ) centered at $i$

$$
\Gamma_{i}^{\prime j}{ }_{k}=O\left(r^{\prime 2}\right), \quad h_{i j}^{\prime}=\delta_{i j}+O\left(r^{\prime 3}\right) .
$$


In these coordinates let $\Psi_{\text {flat }}^{i k} \in C^{\infty}\left(B_{\epsilon} \backslash\{i\}\right)$ be a trace free symmetric and divergence free tensor with respect to the flat metric $\delta_{k l}$,

$$
\delta_{i k} \Psi_{\text {flat }}^{i k}=0, \quad \partial_{i} \Psi_{\text {flat }}^{i k}=0 \text { in } B_{\epsilon} \backslash\{i\},
$$

with

$$
\Psi_{\text {flat }}^{i k}=O\left(r^{\prime-4}\right), \quad \partial \Psi_{\text {flat }}^{i k}=O\left(r^{\prime-5}\right), \quad \partial \partial \Psi_{\text {flat }}^{i k}=O\left(r^{\prime-6}\right) .
$$

All these tensors have been characterized in theorem 14 of [16]. Denote by $Q_{\text {sing }}^{a b}$ the $h^{\prime}$-trace free tensor which is given by

$$
Q_{\text {sing }}^{a b}=\chi_{\epsilon}\left(\Psi_{\text {flat }}^{a b}-\frac{1}{3} h_{c d}^{\prime} \Psi_{\text {flat }}^{c d} h^{\prime a b}\right) .
$$

The tensor $Q_{\text {sing }}^{a b}$ has two important properties. The first one is

$$
D_{a}^{\prime} Q_{\text {sing }}^{a b}=O\left(r^{\prime-2}\right) .
$$

This is a consequence of equations (57) and (58). The second is the following. If the metric $h_{a b}$ admit a conformal Killing vector $\xi^{a}$, then

$$
\int_{\Omega} \xi_{b} D_{a} Q_{\text {sing }}^{a b} d \mu=P^{a} k_{a}+J^{a} S_{a}+A a+\left(P^{c} L_{c}{ }^{a}(i)+Q^{a}\right) q_{a},
$$

where the constants $k^{a}, S^{a}, a$ and $q^{a}$ are the conformal Killing data at $i$ for $\xi^{a}$ given by (16) and the constants $P^{a}, J^{a}, A$ and $Q^{a}$ partly characterize the tensor field $Q_{\text {sing }}^{a b}$ (there exist two free functions that can be freely prescribed in $Q_{\text {sing }}^{a b}$, see [16] for details). Equation (62) is written in terms of the metric $h_{a b}$, it is possible to write it in term to the rescaled metric $h_{a b}^{\prime}$, in this case $L_{a b}^{\prime}(i)=0$.

For the regular part $Q_{r e g}^{a b}$ we will assume that

$$
Q_{\text {reg }}^{a b} \in C^{\infty}(\bar{\Omega} \backslash\{i\}),
$$

and

$$
Q^{a b}=O\left(r^{-1}\right), \quad \partial Q^{a b}=O\left(r^{-2}\right), \quad \partial \partial Q^{a b}=O\left(r^{-3}\right) .
$$

An example is of course $Q_{r e g}^{a b} \in C^{\infty}(\bar{\Omega})$, however the most general condition (64) matches naturally with the fall off of $Q_{\text {sing }}^{a b}$ in the sense that the constant $Q^{a}$ gives the fall of $O\left(r^{-2}\right)$, the constants $A$ and $J^{a}$ the $O\left(r^{-3}\right)$ one and the constants $P^{a}$ the $O\left(r^{-4}\right)$, see [16].

Using the fall off (64) we obtain

$$
\begin{aligned}
\int_{\Omega} \xi_{b} D_{a} Q_{r e g}^{a b} d \mu & =\int_{\partial \Omega} Q_{r e g}^{a b} \xi_{b} \nu_{a} d S-\lim _{\epsilon \rightarrow 0} \int_{\partial B_{\epsilon}} Q_{r e g}^{a b} \xi_{b} n_{a} d S \\
& =\int_{\partial \Omega} Q_{r e g}^{a b} \xi_{b} \nu_{a} d S .
\end{aligned}
$$


We can now prove theorem 2.1

\section{Proof of theorem 2.1}

Proof. Since the conformal factor $\omega_{0}$ defined by (17) is smooth, we can write the equation with respect to the metric $h_{a b}^{\prime}$

$$
\begin{aligned}
\mathbf{L}_{h^{\prime}} w^{\prime a} & =J^{a} \text { in } \Omega, \\
\left(\mathcal{L}_{h^{\prime}} w^{\prime}\right)_{a b} \nu^{\prime a} & =0 \text { on } \partial \Omega .
\end{aligned}
$$

Define $J^{a}=D_{b}^{\prime} Q^{a b}$. By the (61) and (63) we have that $J^{a} \in L^{p}(\Omega)$, with $6 / 5 \leq p<3 / 2$. Then we can apply theorem 4.1. In the presence of conformal Killing vectors the condition $F(\xi)=0$ in theorem 4.1 can be written as the equation (21) using equations (62) and (66). This shows the existence of $w^{a} \in H^{1}(\Omega)$.

The regularity (i.e. $w^{a} \in C^{\infty}(\bar{\Omega} \backslash\{i\})$ and consequently $K^{a b} \in C^{\infty}(\bar{\Omega} \backslash\{i\})$ ) follows directly from the standard elliptic regularity theorems of [3].

Remains to show that $K^{a b}$ satisfies

$$
\left|K^{a b}\right|=O\left(r^{-4}\right) \text {. }
$$

We have that

$$
\left.\left|K^{a b}\right| \leq\left|Q^{a b}\right|+\mid\left(\mathcal{L}_{h^{\prime}} w\right)^{a b}\right) \mid
$$

The first term on the right hand side of this inequality satisfies, by (59) and (64), the estimate (69). We only need to estimate the second term. Let $\Omega^{\prime} \subset \subset \Omega$, we have the following inequality

$$
\left.\sup _{x \in \overline{\Omega^{\prime} \backslash B_{\epsilon}}} \mid\left(\mathcal{L}_{h^{\prime}} w\right)^{a b}\right) \mid \leq C\|w\|_{C^{1}\left(\bar{\Omega}^{\prime} \backslash B_{\epsilon}\right)} \leq C\|w\|_{H^{3}\left(\Omega^{\prime} \backslash B_{\epsilon}\right)},
$$

where the first inequality is obvious and the second is a consequence of the Sobolev imbedding theorem. Using the interior elliptic regularity theorem of [3] we have

$$
\|w\|_{H^{3}\left(\Omega^{\prime} \backslash B_{\epsilon}\right)} \leq C\left(\|J\|_{H^{1}\left(\Omega^{\prime} \backslash B_{\epsilon}\right)}+\|w\|_{L^{2}\left(\Omega^{\prime} \backslash B_{\epsilon}\right)}\right) .
$$

Since $w \in H^{1}(\Omega)$ then $\|w\|_{L^{2}\left(\Omega^{\prime} \backslash B_{\epsilon}\right)}$ is clearly bounded for all $\epsilon$. For the first term in the right hand side of (72) we use the assumptions (59) and (64) to conclude that

$$
\|J\|_{H^{1}\left(\Omega^{\prime} \backslash B_{\epsilon}\right)} \leq \frac{C}{r^{3}},
$$

for every $\epsilon$. Combining the previous inequalities it follows that

$$
\left.\sup _{x \in \overline{\Omega^{\prime} \backslash B_{\epsilon}}} \mid\left(\mathcal{L}_{h^{\prime}} w\right)^{a b}\right) \mid \leq C / r^{3},
$$

for every $\epsilon$. Then, using (170) the desired result follows. 


\section{The Hamiltonian Constraint}

In this section we will prove theorem 2.2. We begin with the item i) of this theorem, that is the existence of the function $\psi_{0}$ defined as a solution of the boundary value problem (22)-(24). This function will play an important role in the non linear existence proof; it is essentially the Green function of the operator $L_{h}$ with the boundary condition (23).

In the following theorem $\delta_{i}$ will denote the Dirac delta distribution with source at $i$.

Theorem 5.1. Assume that the conformal metric $h_{a b}$ satisfies (17) and that $\partial \Omega$ is smooth. Assume also that $R \geq 0$ in $\bar{\Omega}, H \geq 0$ on $\partial \Omega$ and that either $R$ or $H$ is not identically zero. Then, there exist a unique solution $\psi_{0}$ of the boundary value problem (22)-(24). Moreover, $\psi_{0}$ satisfies

$$
L_{h} \psi_{0}=-4 \pi \delta_{i}
$$

in $\Omega, \psi_{0}>0$ in $\bar{\Omega} \backslash\{i\}, \psi_{0} \in C^{\infty}(\bar{\Omega} \backslash\{i\})$ and it has the following form $\psi_{0}=\chi_{\epsilon} / r+u$ with $u \in H^{2}(\Omega)$.

Proof. Observing that $1 / r$ defines a fundamental solution to the flat Laplacian, we obtain

$$
\Delta\left(\frac{\chi_{\epsilon}}{r}\right)=-4 \pi \delta_{i}+\hat{\chi}
$$

where $\hat{\chi}$ is a smooth function on $\bar{\Omega}$ with support in $B_{\epsilon} \backslash B_{\epsilon / 2}$. The ansatz $\psi_{0}=\chi_{\epsilon} / r+u$ translates the original equations into the following equations for $u$

$$
\begin{aligned}
& L_{h} u=-\hat{L}_{h}\left(\frac{\chi_{\epsilon}}{r}\right)-\hat{\chi} \text { in } \Omega, \\
& N_{h} u=0 \text { on } \partial \Omega,
\end{aligned}
$$

where we have defined $\hat{L}_{h}$ by the expansion $L_{h}=\Delta+\hat{L}_{h}$ in normal coordinates centered at $i$. A direct calculation shows that $\hat{L}_{h}\left(\chi_{\epsilon} r^{-1}\right) \in L^{2}(\Omega) \cap$ $C^{\infty}(\bar{\Omega} \backslash\{i\})$. Then, by theorem 3.6 , there exists a unique solution $u \in H^{1}(\Omega)$ of (77) -(78). We can use the standard elliptic regularity theorems to conclude that $u \in H^{2}(\Omega)$, which in particular implies (by the Sobolev imbedding theorem) that $u \in C^{0}(\bar{\Omega})$. We can further use the elliptic regularity in the region $\bar{\Omega} \backslash\{i\}$ to conclude that $u \in C^{\infty}(\bar{\Omega} \backslash\{i\})$.

To show that $\psi_{0}$ is strictly positive, we observe that it is positive near $i$ (because $r^{-1}$ is positive and $u$ is bounded). Take $\epsilon$ small enough such that $\psi_{0}$ is positive on $\partial B_{\epsilon}$. Note that $L_{h} \psi_{0}=0$ and $\psi_{0}$ is smooth in $\bar{\Omega} \backslash B_{\epsilon}$. Let $x_{0} \in \bar{\Omega} \backslash B_{\epsilon}$ the point where $\psi_{0}$ reach its minimum in $\bar{\Omega} \backslash B_{\epsilon}$. Since $L_{h} \psi_{0}=0$, 
we can use the strong maximum principle 3.4 to conclude that either $\psi_{0}$ is constant in $\bar{\Omega} \backslash B_{\epsilon}$ or $x_{0}$ is in the boundary of $\bar{\Omega} \backslash B_{\epsilon}$ and $\psi(x)>\psi\left(x_{0}\right)$ for all $x \in \Omega \backslash B_{\epsilon}$. Since we have assumed that either $R$ or $H$ is not identically zero, $\psi_{0}$ can not be constant. Assume that $\psi_{0}\left(x_{0}\right) \leq 0$. Since $\psi_{0}>0$ on $\partial B_{\epsilon}$, then it follows that $x_{0} \in \partial \Omega$. Consider the function $-\psi_{0}$. We will apply theorem 3.5 on $\bar{\Omega} \backslash B_{\epsilon}$ for this function to get a contradiction. Hypothesis i), ii) and iii) of this theorem are satisfied. Using the boundary condition (78) we have

$$
4 v^{a} D_{a}(-\psi)\left(x_{0}\right)=-H(-\psi)\left(x_{0}\right) \leq 0,
$$

which contradict (43).

We note that theorem 3.5 is essential to prove the strict inequality $\psi_{0}>0$, if we use theorem 3.3 we only get $\psi_{0} \geq 0$.

We treat now the general, non linear, case. Set $\psi=\psi_{0}+u$, then the boundary value problem given by (9), (11) and (14) can be written as follows

$$
\begin{array}{ll}
L_{h} u=f(x, u) & \text { in } \Omega, \\
N_{h} u=\varphi(x, u) & \text { on } \partial \Omega,
\end{array}
$$

where

$$
f(x, u)=-\frac{K^{a b} K_{a b}}{8\left(\psi_{0}+u\right)^{7}}
$$

and

$$
\varphi(x, u)=-\left(\psi_{0}+u\right)^{3} \varphi_{1}+\frac{\varphi_{2}}{\left(\psi_{0}+u\right)^{3}},
$$

where we have used the notation

$$
\Theta_{-}=-\varphi_{1}, \quad K_{a b} \nu^{a} \nu^{b}=\varphi_{2},
$$

to emphasize that the functions $\varphi_{1}$ and $\varphi_{2}$ in the following are arbitrary, non-negative functions which are not necessarily given by by (84).

We first recall some special properties of the functions $f(x, u)$ and $\varphi(x, u)$. The function $f(x, u)$ can be written as

$$
f\left(x, u_{1}\right)-f\left(x, u_{2}\right)=\left(u_{1}-u_{2}\right) \hat{f}\left(x, u_{1}, u_{2}\right),
$$

where

$$
\hat{f}\left(x, u_{1}, u_{2}\right)=\frac{K^{a b} K_{a b}}{8} \sum_{j=0}^{6}\left(\psi_{0}+u_{1}\right)^{j-7}\left(\psi_{0}+u_{2}\right)^{-1-j} .
$$

Clearly $\hat{f}\left(x, u_{1}, u_{2}\right) \geq 0$ for any $u_{1}, u_{2} \geq 0$. Also, for any $u \geq 0$ we have

$$
0 \geq f(x, u) \geq f(x, 0)
$$


For $\varphi(x, u)$ we have a similar expression

$$
\varphi\left(x, u_{1}\right)-\varphi\left(x, u_{2}\right)=\left(u_{2}-u_{1}\right) \hat{\varphi}\left(x, u_{1}, u_{2}\right)
$$

where

$$
\hat{\varphi}\left(x, u_{1}, u_{2}\right)=\sum_{j=0}^{2} \varphi_{1}\left(\psi_{0}+u_{1}\right)^{2-j}\left(\psi_{0}+u_{2}\right)^{j}+\varphi_{2}\left(\psi_{0}+u_{1}\right)^{-j-1}\left(\psi_{0}+u_{2}\right)^{-3-j}
$$

If we assume $\varphi_{1}, \varphi_{2} \geq 0$, then we have that $\hat{\varphi}\left(x, u_{1}, u_{2}\right) \geq 0$ for any $u_{1}, u_{2} \geq 0$. However, in contrast to $f(x, 0)$, the function $\varphi(x, 0)$ has, in principle, no definite sign.

We define $u_{+}$as a solution of the following linear problem

$$
\begin{aligned}
& L_{h} u_{+}=f(x, 0) \text { in } \Omega, \\
& N_{h} u_{+}=\varphi_{2} \psi_{0}^{-3} \text { on } \partial \Omega .
\end{aligned}
$$

If $\varphi_{2} \geq 0$ and $f(x, 0) \in L^{2}(\Omega)$, then, by theorem 3.6, there exist a unique solution $u_{+} \geq 0$ of (90)-(91). Note that the function $\psi_{1}$ defined by (25) $-(27)$ is given by $\psi_{1}=\psi_{0}+u_{+}$. The following theorem will prove items ii) and iii) of theorem 2.2 .

Theorem 5.2. Assume that the hypothesis of theorem 5.1 are in force. In addition, we assume that $\varphi_{1}, \varphi_{2}$ are smooth function on $\partial \Omega$ which satisfy $\varphi_{1}, \varphi_{2} \geq 0, K_{a b} K^{a b} \psi_{0}^{-7} \in L^{2}(\Omega)$ and $\varphi\left(x, u_{+}\right) \geq 0$ where $u_{+}$is the unique solution of (90)-(91). Then, there exist a unique positive solution $u \in H^{2}(\Omega) \cap C^{\infty}(\bar{\Omega} \backslash\{i\})$ of the boundary value problem (80)-(81). Moreover, $0 \leq u \leq u_{+}$.

Proof. The proof is based on the Schauder fixed point theorem 3.9. Define $B \subset C^{0}(\bar{\Omega})$ as

$$
B=\left\{u \in C^{0}(\bar{\Omega}): 0 \leq u \leq u_{+}\right\} .
$$

Clearly $B$ is closed and convex subset of the Banach space $C^{0}(\bar{\Omega})$. Let $w \in B$, we define the map $T(w)=u$ as follows. Let $u$ be the unique solution given by theorem 3.6. of the following linear boundary value problem

$$
\begin{aligned}
& L_{h} u=f(x, w) \text { in } \Omega \\
& N_{h} u=\varphi(x, w) \text { on } \partial \Omega .
\end{aligned}
$$

Using equation (88), (85), the maximum principle 3.3 and the condition $\varphi\left(x, u_{+}\right) \geq 0$ one shows that $T(B) \subset B$. Using the elliptic estimate 3.7 we have that $u \in C^{\alpha}(\bar{\Omega})$, and hence the image of $T(B)$ is precompact because 
$C^{\alpha}(\bar{\Omega})$ is compactly imbedded in $C^{0}(\bar{\Omega})$. It is also clear that $T$ is continuous. Then, by the Schauder fixed point theorem 3.9. there exist a fixed point $T(u)=u$ with $u \in C^{0}(\bar{\Omega})$. The standard elliptic regularity implies that in fact $u \in H^{2}(\Omega) \cap C^{\infty}(\bar{\Omega} \backslash\{i\})$. This finishes the existence part.

To prove uniqueness, let assume that there exist two solutions $u_{1}$ and $u_{2}$, the difference $u_{1}-u_{2}$ will satisfy the equations

$$
\begin{aligned}
& L_{h}\left(u_{1}-u_{2}\right)=\left(u_{1}-u_{2}\right) \hat{f}\left(x, u_{1}, u_{2}\right) \\
& N_{h}\left(u_{1}-u_{2}\right)=\left(u_{2}-u_{1}\right) \hat{\varphi}\left(x, u_{1}, u_{2}\right)
\end{aligned}
$$

where $\hat{f}$ and $\hat{\varphi}$ are given by (86) and (89) respectively. Assume that $u_{1} \neq u_{2}$ in some set. Since $u_{1}$ and $u_{2}$ are continuous functions, we can find a set $\Omega^{\prime} \subset \bar{\Omega}$ such that $u_{1}>u_{2}$ in $\Omega^{\prime}$ (we change $u_{1}$ by $u_{2}$ if necessary), $u_{1}-u_{2}=0$ on $\partial \Omega^{\prime}-\Gamma$, where $\Gamma$ denotes the portion of $\partial \Omega^{\prime}$ contained in $\partial \Omega$ and $u_{1}-u_{2} \geq 0$ on $\Gamma$. By (95) we have $L_{h}\left(u_{1}-u_{2}\right) \geq 0$. $u_{1}-u_{2}$ can not be a positive constant in $\Omega^{\prime}$ because $\hat{f} \geq 0$ and $R \geq 0$. Then by the strong maximum principle there exist $x_{0} \in \partial \Omega^{\prime}$ such that $\left(u_{1}-u_{2}\right)\left(x_{0}\right)>\left(u_{1}-u_{2}\right)(x)$ for all $x \in \Omega^{\prime}$. The point $x_{0}$ can not be on $\partial \Omega^{\prime}-\Gamma$ because there we have $u_{1}-u_{2}=0$ and this will contradict $u_{1}>u_{2}$. Then $x_{0} \in \Gamma$. Using the boundary condition (96) we get

$$
4 \nu^{a} D_{a}\left(u_{1}-u_{2}\right)\left(x_{0}\right) \leq-H\left(u_{1}-u_{2}\right)\left(x_{0}\right) \leq 0 .
$$

We use theorem 3.5 to get a contradiction.

Note that $\varphi\left(x, u_{+}\right) \geq 0$ is precisely condition (29). To finish the proof of the item iii) of theorem 2.2 it remains only to prove the inequality (30). Using equations (12)-(13) we obtain

$$
\Theta_{-}-\Theta_{+}=2 N_{h} \psi=2 \varphi(x, u) \geq 2 \varphi\left(x, u_{+}\right) \geq 0 .
$$

Using the sub and super solution method, boundary value problem of the form (95)-(96) has been studied in [4] under stronger regularity assumptions on the coefficients (see also [34]). In [27] a related equation has been also studied using a variational approach.

\section{Acknowledgments}

It is a pleasure to thank H. Friedrich, M. Mars and B. Schmidt for illuminating discussions. I would also like to thank the organizers of the "Penrose inequalities workshop", R. Beig, P. Chrusciel and W. Simon, and the friendly hospitality of the Erwin Schrödinger Institute for Mathematical Physics (ESI), where part of this work was done.

This work has been supported by the Sonderforschungsbereich SFB/TR7 of the Deutsche Forschungsgemeinschaft. 


\section{References}

[1] R. A. Adams. Sobolev Spaces. Academic Press, New York, 1975.

[2] S. Agmon. Lectures on Elliptic Boundary Value Problems, volume 2 of Van Nostrand Mathematical Studies. D. van Nostrand Company, Inc, 1965.

[3] S. Agmon, A. Douglis, and L. Niremberg. Estimates near the boundary for solutions of elliptic partial differential equations satisfying general boundary conditions. II. Comm. Pure App. Math., 17:35-92, 1964.

[4] H. Amann. On the existence of positive solutions of nonlinear elliptic boundary value problems. Indiana Univ. Math. J., 21:125-146, 1971.

[5] R. Beig and N. O. Murchadha. Trapped surfaces in vacuum spacetimes. Class. Quantum. Grav., 11(2):419-430, 1994.

[6] R. Beig and N. O. Murchadha. The momentum constraints of general relativity and spatial conformal isometries. Commun. Math. Phys., 176(3):723-738, 1996.

[7] M. Cantor and D. Brill. The Laplacian on asymptotically flat manifolds and the specification of scalar curvature. Compositio Mathematica, 43(3):317-330, 1981.

[8] Y.-Z. Chen and L.-C. Wu. Second order Elliptic Equations and Elliptic Systems, volume 174 of Translations of mathematical monographs. American Mathematical Society, 1998.

[9] Y. Choquet-Bruhat, J. Isenberg, and J. W. York, Jr. Einstein constraint on asymptotically euclidean manifolds. Phys. Rev. D, 61:084034, 1999, gr-qc/9906095.

[10] Y. Choquet-Bruhat and J. W. York, Jr. The Cauchy problem. In A. Held, editor, General Relativity and Gravitation, volume 1, pages 99-172. Plenum, New York, 1980.

[11] G. B. Cook. Initial data for numerical Relativity. Living Rev. Relativity, 3(5), 2001. http://www.livingreviews.org/Articles/Volume3/2000-5cook/

[12] S. Dain. Initial data for stationary space-time near space-like infinity. Class. Quantum. Grav., 18(20):4329-4338, 2001, gr-qc/0107018. 
[13] S. Dain. Asymptotically flat and regular Cauchy data. In J. Frauendiener and H. Friedrich, editors, The Conformal Structure of Spacetimes: Geometry, Analysis, Numerics, volume 604 of Lecture Notes in Physics. Springer, 2002, gr-qc/0203021.

[14] S. Dain. Black hole interaction energy. Phys. Rev. D, 66(8):084019, 2002, gr-qc/0207090

[15] S. Dain. Initial data for black hole collisions. In A. Lobo, F. Fayos, J. Garriga, E. Gaztañaga, and E. Verdaguer, editors, Proceedings of the Spanish Relativity Meeting Gravitation and Cosmology ERE - 2002, pages 183-187. Universitat de Barcelona, 2003, gr-qc/0212039.

[16] S. Dain and H. Friedrich. Asymptotically flat initial data with prescribed regularity. Commun. Math. Phys., 222(3):569-609, 2001, gr-qc/0102047.

[17] S. Dain and G. Nagy. Initial data for fluid bodies in general relativity. Phys. Rev. D, 65(8):084020, 2002, gr-qc/0201091.

[18] J. F. Escobar. The Yamabe problem on manifolds with boundary. $J$. Differential Geom., 35(1):21-84, 1992.

[19] G. B. Folland. Introduction to Partial Differential Equation. Princeton University Press, Princeton, NY, 1995.

[20] H. Friedrich. On static and radiative space-time. Commun. Math. Phys., 119:51-73, 1988.

[21] H. Friedrich. Gravitational fields near space-like and null infinity. $J$. Geom. Phys., 24:83-163, 1998.

[22] D. Gannon. Singularities in nonsimply connected space-times. J. Math. Phys., 16(12):2364-2367, 1975.

[23] M. Giaquinta. Introduction to Regularity Theory for Nonlinear Elliptic Systems. Lectures in Mathematics (ETH Zürich). Birkhäuser Verlag, Basel, Boston, Berlin, 1993.

[24] D. Gilbarg and N. S. Trudinger. Elliptic Partial Differential Equations of Second Order. Springer-Verlag, Berlin, 1983.

[25] Q. Han and F. Lin. Elliptic Partial Differential Equations. Courant Lectures Notes in Mathematics. Courant Institut of Mathematical Physics, New York, 1997. 
[26] S. W. Hawking and G. F. R. Ellis. The large scale structure of spacetime. Cambridge University Press, Cambridge, 1973.

[27] M. Herzlich. A Penrose-like inequality for the mass of Riemannian asymptotically flat manifolds. Commun. Math. Phys., 188:121-133, 1997.

[28] S. Husa. Initial data for general relativity containing a marginally outer trapped torus. Phys. Rev., D54:7311-7321, 1996, gr-qc/9606042.

[29] S. Husa. Asymptotically flat initial data for gravitational wave spacetimes, conformal compactification and conformal symmetry. $\mathrm{PhD}$ thesis, University of Vienna, 1998.

[30] O. A. Ladyzhenskaya and N. N. Ural'tseva. Linear and Quasilinear Elliptic Equations. Academic Press, New York, 1968.

[31] J. E. Marsden and T. J. Hughes. Mathematical Foundations of Elasticity. Prentice-Hall civil engineering and engineering mechanics series. Prentice Hall, Inc., Englewood Cliffs, New Jersey, 1983.

[32] D. Maxwell. Solutions of the Einstein constraint equations with apparent horizon boundary. 2003, gr-qc/0307117.

[33] C. W. Misner, K. S. Thorne, and J. A. Wheeler. Gravitation. W.H. Freeman and Company, San Francisco, 1973.

[34] C.-V. Pao. Nonlinear Parabolic and Elliptic Equations. Plenum Press, New York, 1992.

[35] R. Penrose. Gravitational collapse and space-time singularities. Phys. Rev. Lett., 14:57-59, 1965.

[36] J. Thornburg. Coordinates and boundary conditions for the general relativistic initial data problem. Classical and Quantum Gravity, 4(5):11191131, 1987.

[37] N. S. Trudinger. Maximum principles for linear, non-uniformly elliptic operators with measurable coefficients. Math. Z., 156(3):291-301, 1977.

[38] R. M. Wald. General Relativity. The University of Chicago Press, Chicago, 1984.

[39] K. Yano. The theory of Lie derivatives and its applications. North Holland, Amsterdam, 1957. 
[40] J. W. York, Jr. Conformally invariant orthogonal decomposition of symmetric tensor on Riemannian manifolds and the initial-value problem of general relativity. J. Math. Phys., 14(4):456-464, 1973. 